\title{
THE VELOCITY PATTERN OF WEAK SOLAR MAGNETIC FIELDS
}

\author{
H. WANG and H. ZIRIN \\ Big Bear Solar Observatory, California Institute of Technology, Pasadena, CA 91125, U.S.A.
}

(Received 23 October, 1987)

\begin{abstract}
We have measured the proper motion of magnetic elements on the quiet Sun by means of local correlation tracking. The existence of a pattern in the intranetwork (IN) flow is confirmed. This velocity field is consistent with the direct Doppler measurement of the horizontal component of the supergranular velocity field. The IN elements generally move toward the network boundaries. By tracking test points we confirm that the magnetic elements converge in areas corresponding to the magnetic network. But because the IN elements are of random polarity, they cannot contribute to the growth or maintenance of the magnetic network.

By calculating the cross correlation between the magnetogram and Dopplergram, we confirm that the supergranule boundaries and the magnetic network are roughly correlated.
\end{abstract}

\section{Introduction}

The supergranule velocity pattern was discovered by Hart $(1954,1956)$. It was first named and studied extensively by Leighton et al. (1962) and Simon and Leighton (1964). They described this phenomena as the cellular convective motion. They also found a strong spatial correlation between the supergranule boundaries and several other features, e.g., chromospheric $\mathrm{CaK}, \mathrm{H} \alpha$ networks and the network magnetic fields. Some theoretical work has been done to study the interaction of isolated magnetic flux tubes or uniformly distributed magnetic fields with convection in the Sun (Clark, 1968; Meyer, Schmidt, and Simon, 1979; Galloway and Weiss, 1981; Spruit, 1981; Parker, 1982; Schmidt, Simon, and Weiss, 1985). On theoretical grounds these authors conclude that: uniformly distributed magnetic fields should be swept to the boundaries of the supergranules; flux tubes of magnitude $\leq 10^{19} \mathrm{Mx}$ should follow the motion of convection pattern, while larger flux tubes would respond to the magnetic buoyancy force.

The intranetwork (IN) magnetic fields were first discovered by Livingston and Harvey (1975), who found the following characteristics: mixed polarity; flux elements as small as $5 \times 10^{16} \mathrm{Mx}$; lifetime 5 to $90 \mathrm{~min}$ and possible radial proper motion. This paper was only an abstract with limited information.

Following the recent improvements in the videomagnetograph (VMG) system at the Big Bear Solar Observatory, a number of studies of the small-scale quiet Sun magnetic fields have been carried out, including network and IN fields, ephemeral regions, and cancelling features (Martin, 1983; Wang, Zirin, and Shi, 1985; Martin, Livi, and Wang, 1985; Livi, Wang, and Martin, 1985; Chou and Wang, 1987; Zirin, 1985, 1987). Zirin (1985) studied the IN elements and their possible role in replenishing elements of the quiet network. He found a mean velocity of $0.35 \mathrm{~km} \mathrm{~s}^{-1}$ for elements lasting over 90 
min. Martin (1987) proposed the term Intranetwork Flow (INF) for the possible systematic motion of IN elements. In general the BBSO data show the IN elements to move rectilinearly and live until they reach the network boundaries.

Title et al. (1987) used the SOUP instrument to obtain white light movies of the solar photosphere free of atmospheric seeing and distortion. These data permitted measurement of the supergranular flow by correlation tracking of individual granules.

In the present work we use reregistered and processed versions of Big Bear VMG movies to measure the INF by correlation tracking of magnetic elements. If we assume that the motion of the IN elements is due to local flows, then these relatively long-lived and fast moving features make excellent tracers of the flow. The cross-correlation curve between magnetic and velocity fields shows a rough relationship between the supergranule boundaries and the network magnetic fields. Local correlation tracking of IN elements confirms the cellular INF pattern.

\section{Instrument and Observation}

Observations were made on June 22, 1985 and October 15, 1985 with the VMG system at BBSO (Mosher, 1976; Zirin, 1985). A series of VMG images of October 15, 1985 are shown in Figure 1. The contour-like curves are caused by wrapping the signal around to the opposite sign when the 12-bit memory is filled. Magnetograms were obtained by differencing 4096 pairs of Zeeman frames in the 12-bit memory and outputting the most significant 8 bits. Because of interpolation of $\mathrm{H} \alpha$ frames with the same 25-cm telescope, the interval between frames was 8-10 min (presently about $150 \mathrm{~s}$ are required). Observations were carried out for 10 to $12 \mathrm{hr}$ per day and all images were stored to both film and magnetic tape.

During the October 1985 run, simultaneous magnetograms and Dopplergrams were made at the National Solar Observatory at Kitt Peak. Because observations of longitudinal magnetic fields are best made at the disk center while the supergranular horizontal velocity is best observed near the limb, we chose a target region at a heliocentric angle of $30 \mathrm{deg}$. The vacuum tower and 512-channel magnetograph system at Kitt Peak have been described by Livingston et al. (1976a, b). To correct for the 5-min oscillation, the Kitt Peak Dopplergrams were taken at a rate of 150 s per scan. The scan area is $256^{\prime \prime}$ by 256", similar to the field of view at Big Bear. With the aid of Kitt Peak magnetograms, we can compare the Big Bear magnetograms and Kitt Peak Dopplergrams without difficulty.

\section{Data Reduction}

(a) Registration: the Big Bear magnetograms were reregistered with the Megavision Image Processor at Caltech. The software uses the maximum correlation principle. A few bad images were removed; solar rotation and guiding fluctuation were removed.

(b) Unwrapping and calibration: the contour-like wraps used to extend our dynamic range were removed by software, and the magnetograms were calibrated by using measurements of solar rotation (Shi, Wang, and Patterson, 1985). 


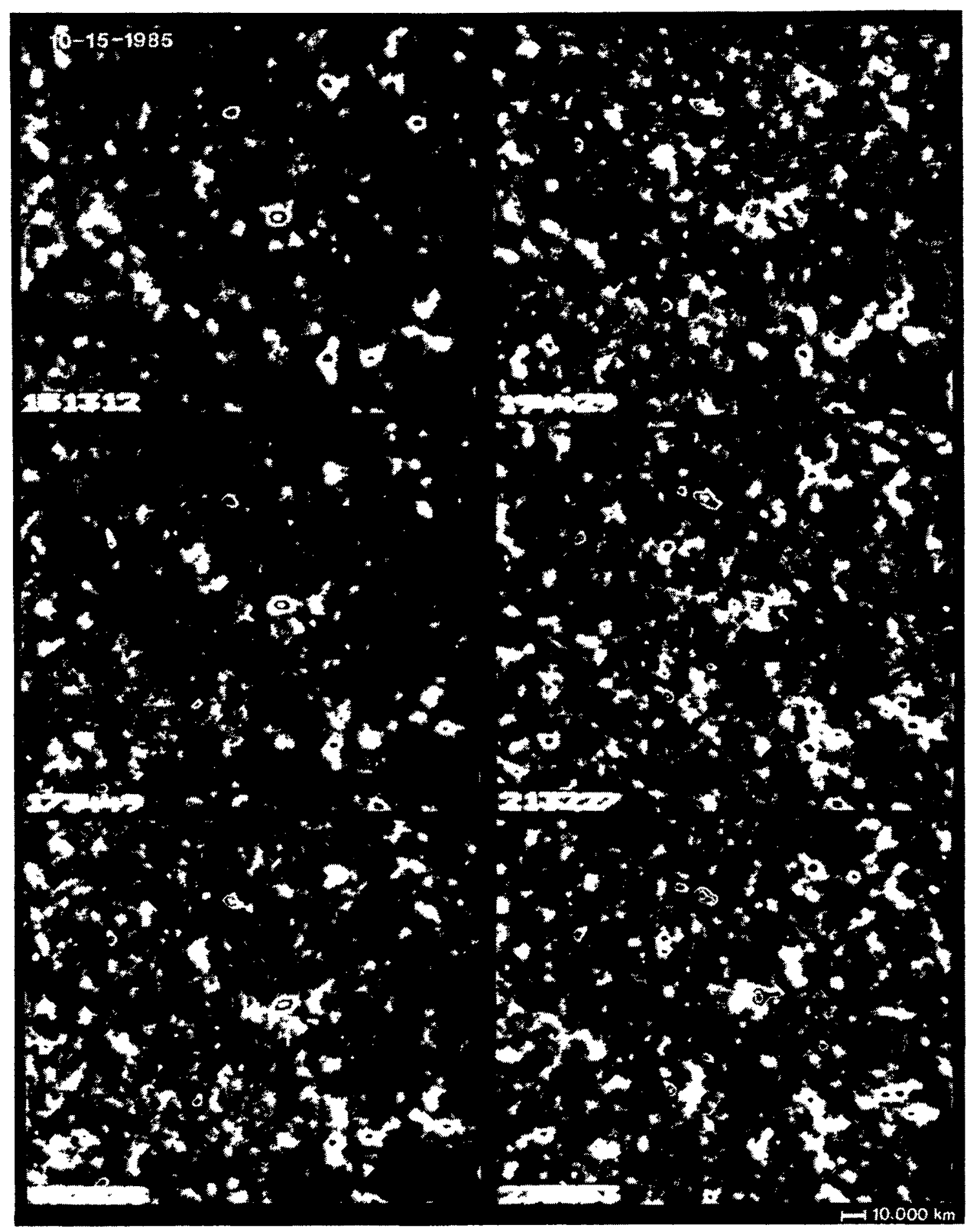

Fig. 1. The time sequence Big Bear magnetograms made on October 15, 1985.

(c) Running means: four consecutive images were averaged, increasing the signal-tonoise ratio by a factor of 1.7 . The sensitivity of the magnetic field measurement is increased from 5 to $3 \mathrm{G}$ over the $2 \times 2$ arc sec resolution element. Each averaged frame is equivalent to a 25-min, 16000-frame integration magnetogram. We feel that the averaging of 4 images is an upper limit for the 1985 data. If we average more frames, the evolution and the motion of intranetwork magnetic fields would smear the image. 
(d) Correction for Dopplergrams: the details of this procedure are described in another paper (Wang and Zirin, 1988a). We made 4-image averages and performed spatial Fourier filtering to remove the 5-min oscillation and other small-scale velocity patterns such as the granulation.

\section{Cross-Correlation of Magnetic and Velocity Fields}

Previous observational and theoretical work suggested that the magnetic fields are swept to the boundaries of supergranules and form the magnetic network. Simon and Leighton (1964) used a simple sinusoidal model for the horizontal component of the supergranule velocity fields:

$$
F_{h}(\rho)=V_{h} \sin (2 \pi \rho / L)
$$

where $L$ is the cell diameter, $\rho$ is the radius vector of a plane-polar coordinate system in the solar surface with origin at the center of the cell. Giovanelli (1980) concluded that the vertical velocity of supergranular flow at the photospheric level is $\leq 0.01 \mathrm{~km} \mathrm{~s}^{-1}$. Our observations confirm this conclusion (Wang and Zirin, 1988a). Also we confirm

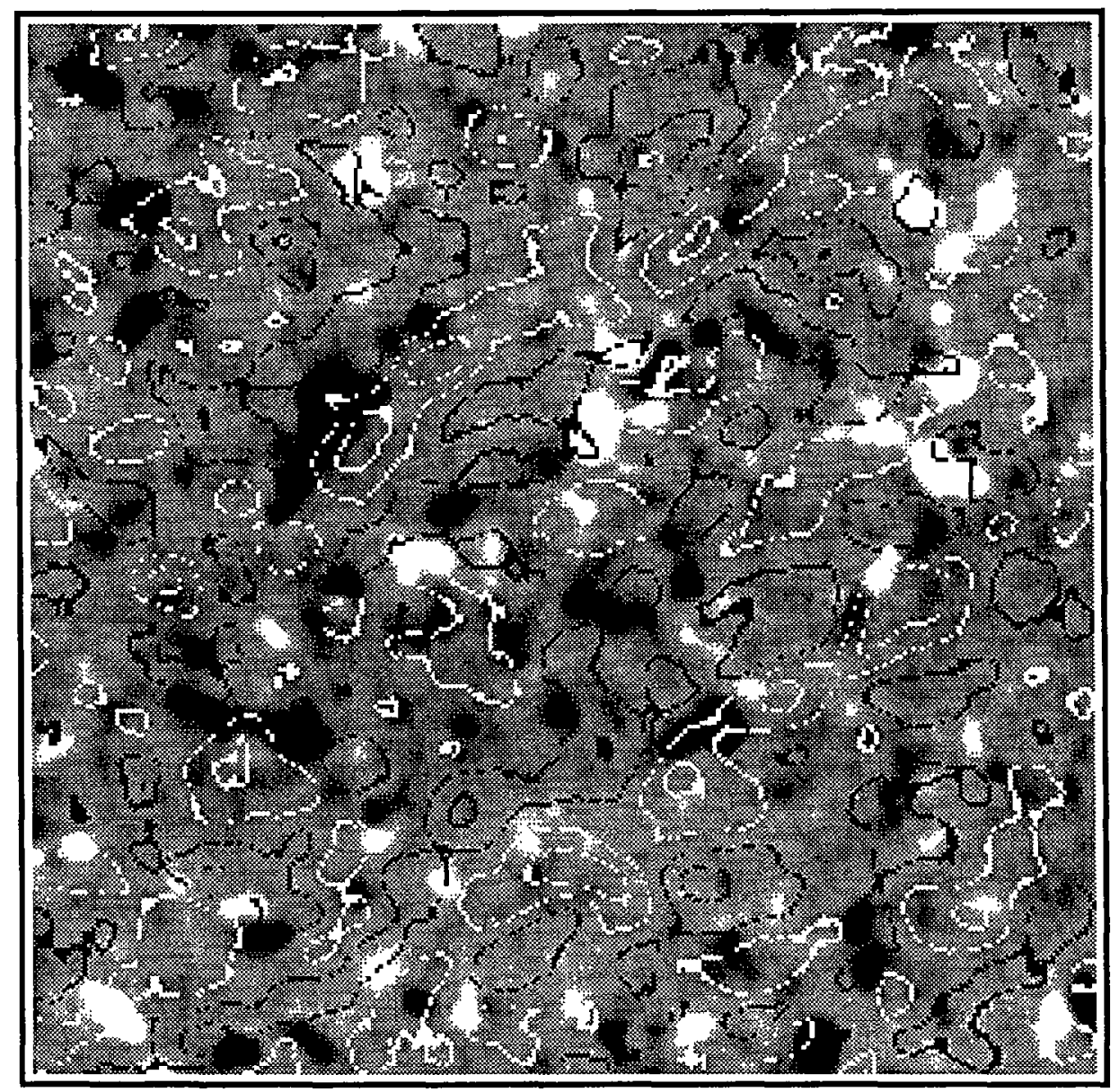

Fig. 2a. A Kitt Peak magnetogram and the simultaneous Dopplergram. The contours represent the velocity fields and the gray scales represent the magnetic fields. They were made at 17:30 UT, October 15, 1985. 


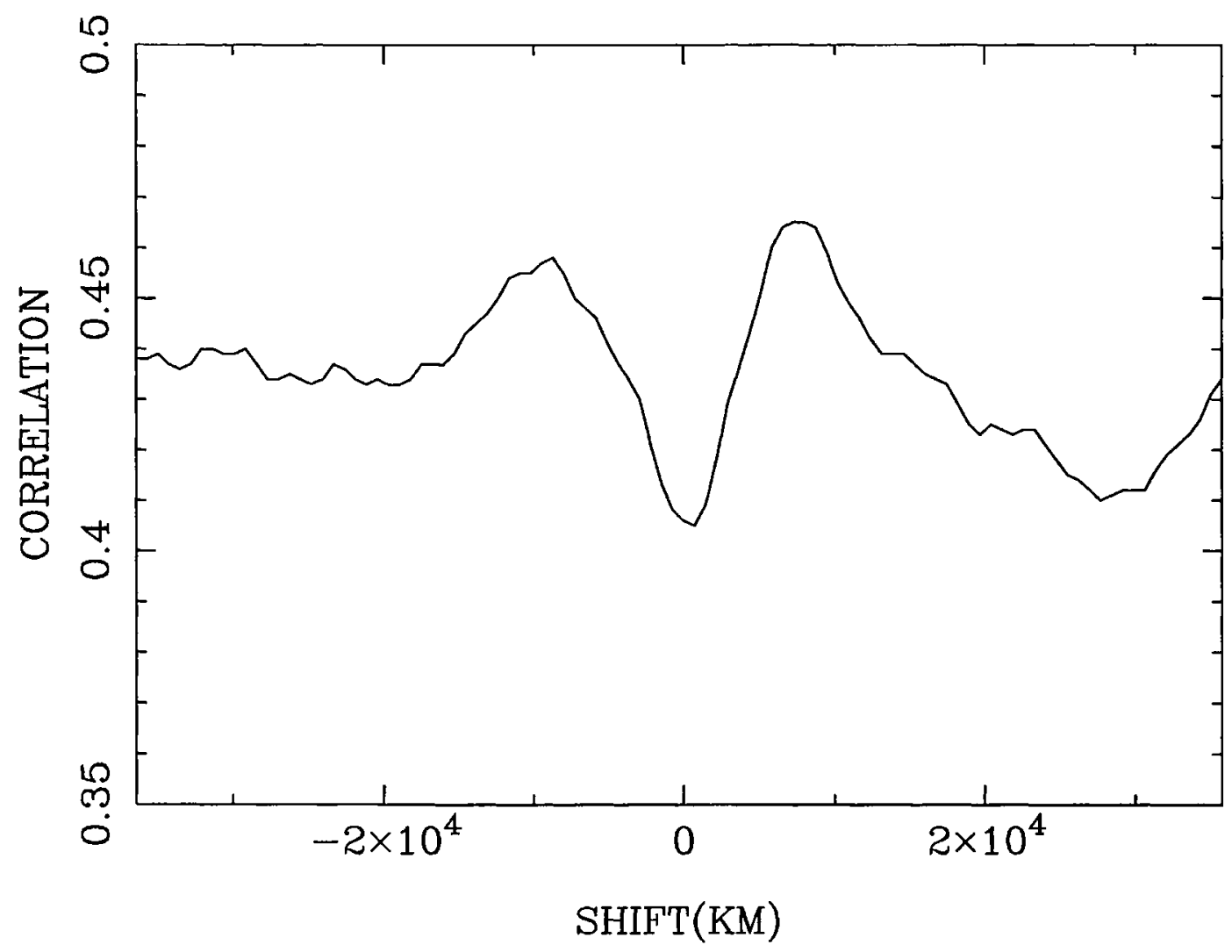

Fig. 2b. Velocity and magnetic fields correlation curve.

Leighton's result for the horizontal component of the supergranule velocity, i.e., the mean speed is $0.3 \mathrm{~km} \mathrm{~s}^{-1}$ with a peak velocity of $0.5 \mathrm{~km} \mathrm{~s}^{-1}$ (Leighton, Noyes, and Simon, 1962). So the horizontal velocity dominates the supergranular velocity fields. At the supergranule boundary, $\rho=L / 2$, i.e., $F_{h}(\rho)=0$. If the strong magnetic elements are located at the boundary of the supergranular flow pattern, the distribution of magnetic fields would have a peak at $\rho=L / 2$, and the cross-correlation of magnetic field and velocity field would have a dip at $\Delta X=0$, where $\Delta X$ is the relative shift between the two images.

In order quantitatively to describe the correlation between the supergranule boundary and the magnetic network, we calculated the cross-correlation between the magnetograms and Dopplergrams. Figure 2(a) shows a Kitt Peak magnetogram: Dopplergram pair made on October 15,1985 . The white and dark contours represent the receding and approaching motion, respectively, while the light and dark gray levels represent the magnetic fields with positive and negative polarity. First, we converted the two images to absolute values, then we calculated the $B-V$ cross-correlation for various relative shifts $X$ in the direction (Figure 3), normal to the solar limb. This cross-correlation is plotted in Figure 2(b). A 13\% dip in the cross-correlation coefficient appears at $\Delta X=0$, indicating that the magnetic network is roughly related to the supergranular flow. The curve has a periodic property; the distance between two maxima is the average radius of the supergranule cells. The distance is about $17000 \mathrm{~km}$ giving an average cell diameter 


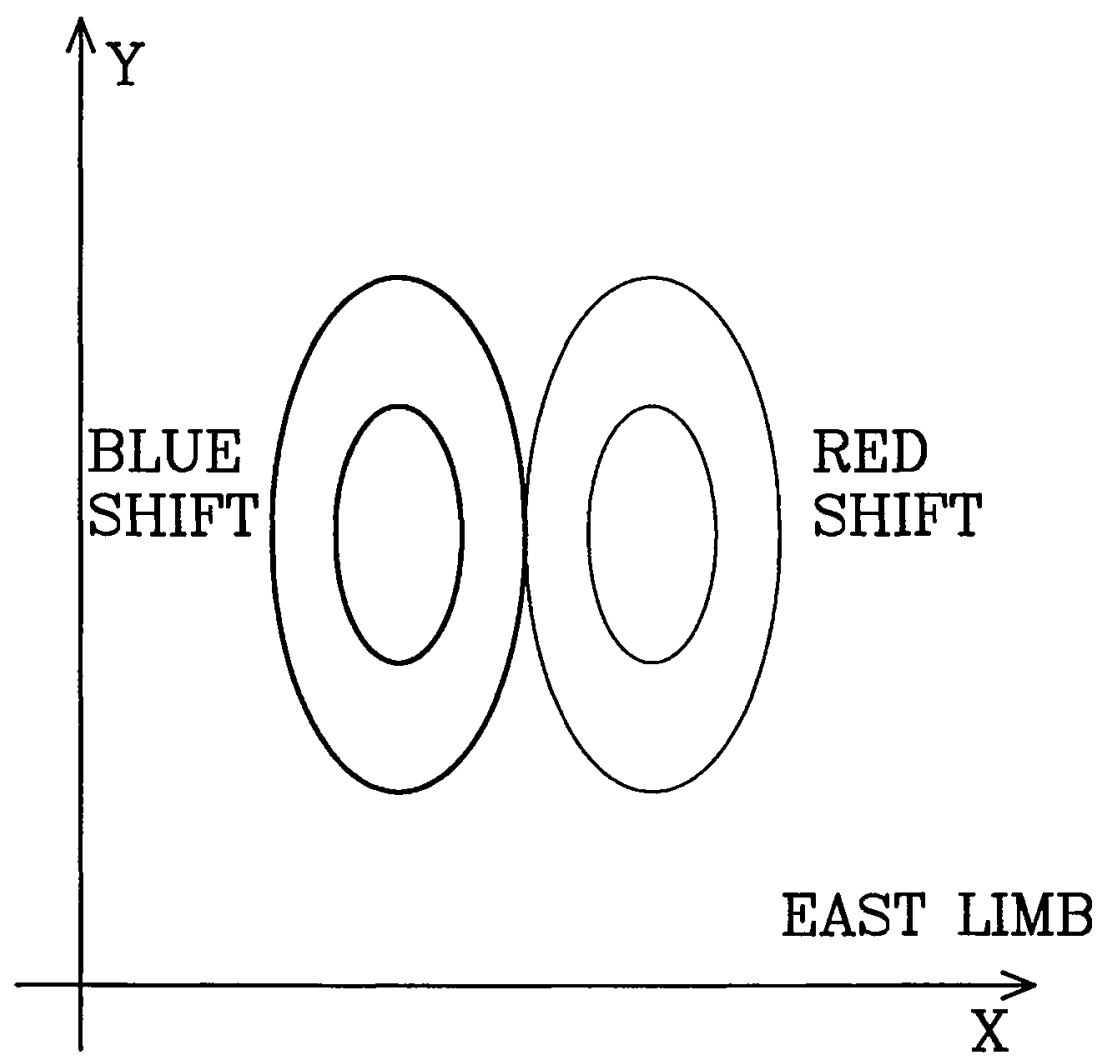

Fig. 3. Schematic map to explain the geometric direction of the Dopplergram.

of $34000 \mathrm{~km}$, consistent with Leighton's early result (Leighton, Noyes, and Simon, 962; Simon and Leighton, 1964).

\section{Correlation Tracking of the Intranetwork Flow}

Figure 4 shows the contour map of a Big Bear magnetogram taken at $19: 47$ on the same day. The lighter contours represent negative polarity, and the darker, positive. Figure 5 is the local correlation tracking map for the same day, where arrows indicate the local motion derived by moving each window until the correlation between frames is maximized. It is the average map for the whole day, but 2- or 3- $\mathrm{hr}$ averaging gives quite similar results. Figure 4 covers about $250^{\prime \prime} \times 250^{\prime \prime}$, so there are about 30 supergranule cell inside the field of view. Each image is divided into many small windows; each window represents an indicator of the local proper motion. The size of each window should be bigger than that of individual magnetic elements. A value of about 7 arc sec was derived from the FWHM of the autocorrelation (Wang and Zirin, 1988b). That size only applies to the network elements; the IN elements are 2 arc sec or smaller. Since the flow pattern we are studying is related to the supergranulation, the window size should be substantially smaller than the size of a supergranule, about $45 \mathrm{arc} \mathrm{sec}$. We choose $10^{\prime \prime}$ as the window size.

Tracking results for two groups of pairs, separated by 30 and $60 \mathrm{~min}$, respectively, 


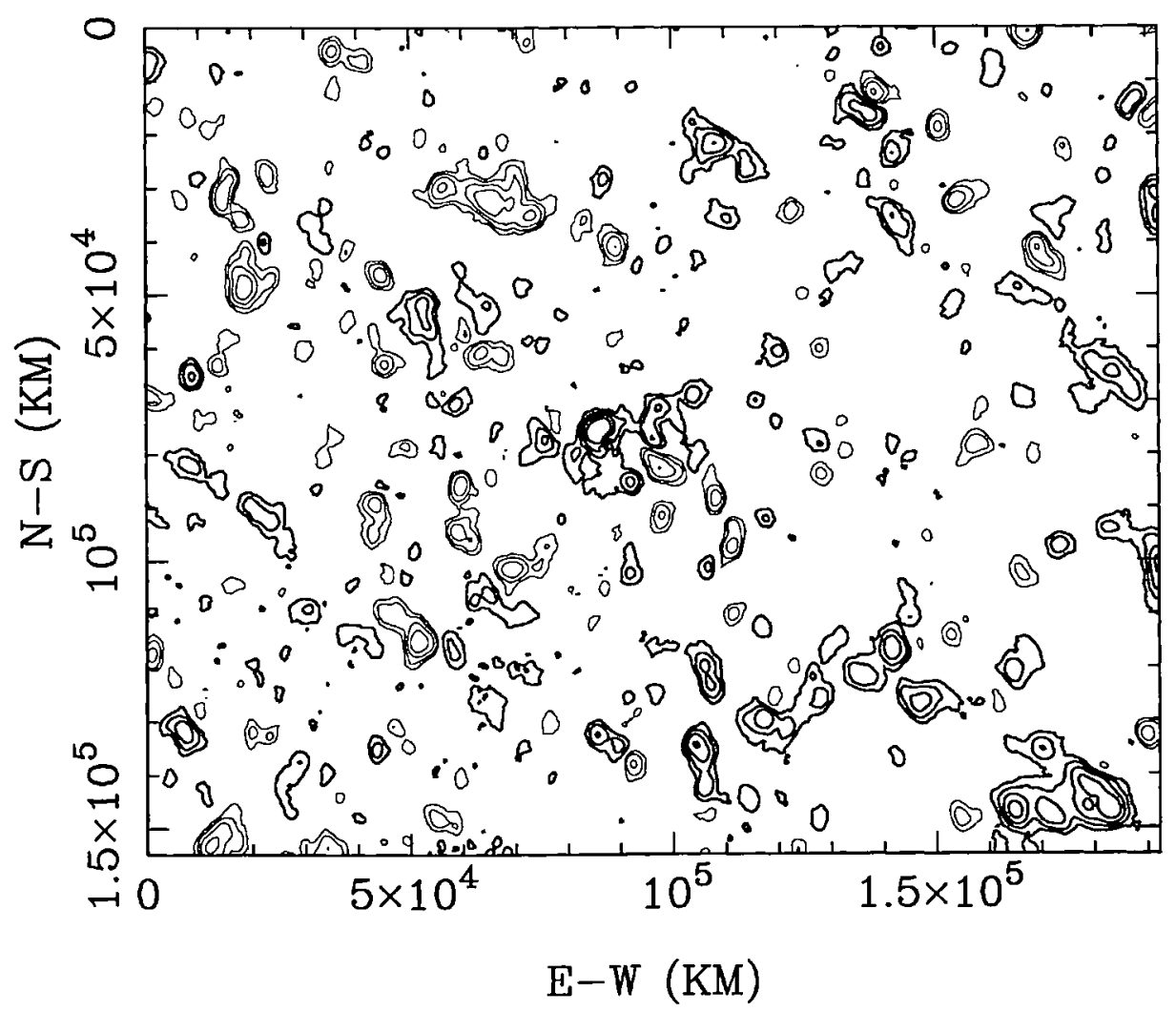

Fig. 4. Contour map for the magnetogram made on October $15,1985$.

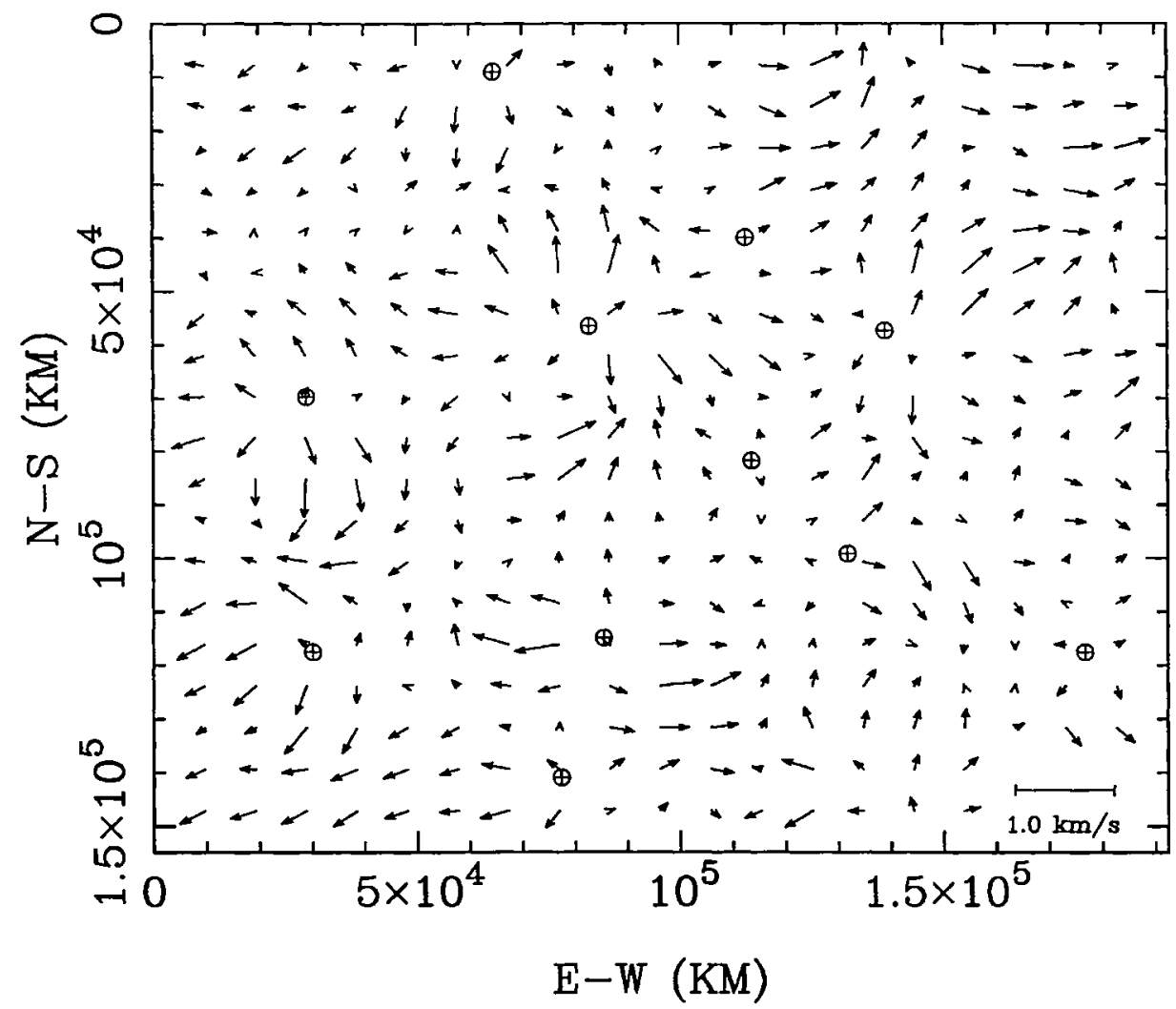

Fig. 5. The local correlation tracking map; the arrows represent the local proper motion velocity, their length is proportional to the speed. 
were analyzed. The results for the two time intervals were nearly the same. Figure 4 is the average of these two groups.

Figure 5 shows the resulting map of the proper motion of magnetic elements. We see a diverging flow pattern in a number of locations, some of which are marked by $\oplus$. Comparison with the movie shows that the map really presents the true motion. In Figure 6, we plot the magnetogram (Figure 4) and the tracking map (Figure 5) together. The stronger magnetic elements outline the network and almost every diverging point is located at the center of a network cell, a place of low flux. Of course, no matter which direction the IN elements move they will be pointed at the network, but the map shows that almost all arrows point to the nearest network element.

Figure 7 gives the comparison between the direct Doppler measurement of supergranule velocity and the correlation tracking for October 15,1985 . We use the contours to represent the Doppler measurement. The east limb of the Sun is at the upper-right corner of the map. Darker contours are approaching motion, lighter contours are receding motion. So one pair of contours with a lighter contour at the upper right and darker one at lower left represents a single supergranule cell. The line which divides the lighter and darker contours from upper right to lower left passes through the center of a supergranule, and the opposite pair passes through the boundary. In this figure, we can see that most of the diverging points are located at the center of a supergranule cell, the converging points are located at a boundary, and the proper motion of the magnetic

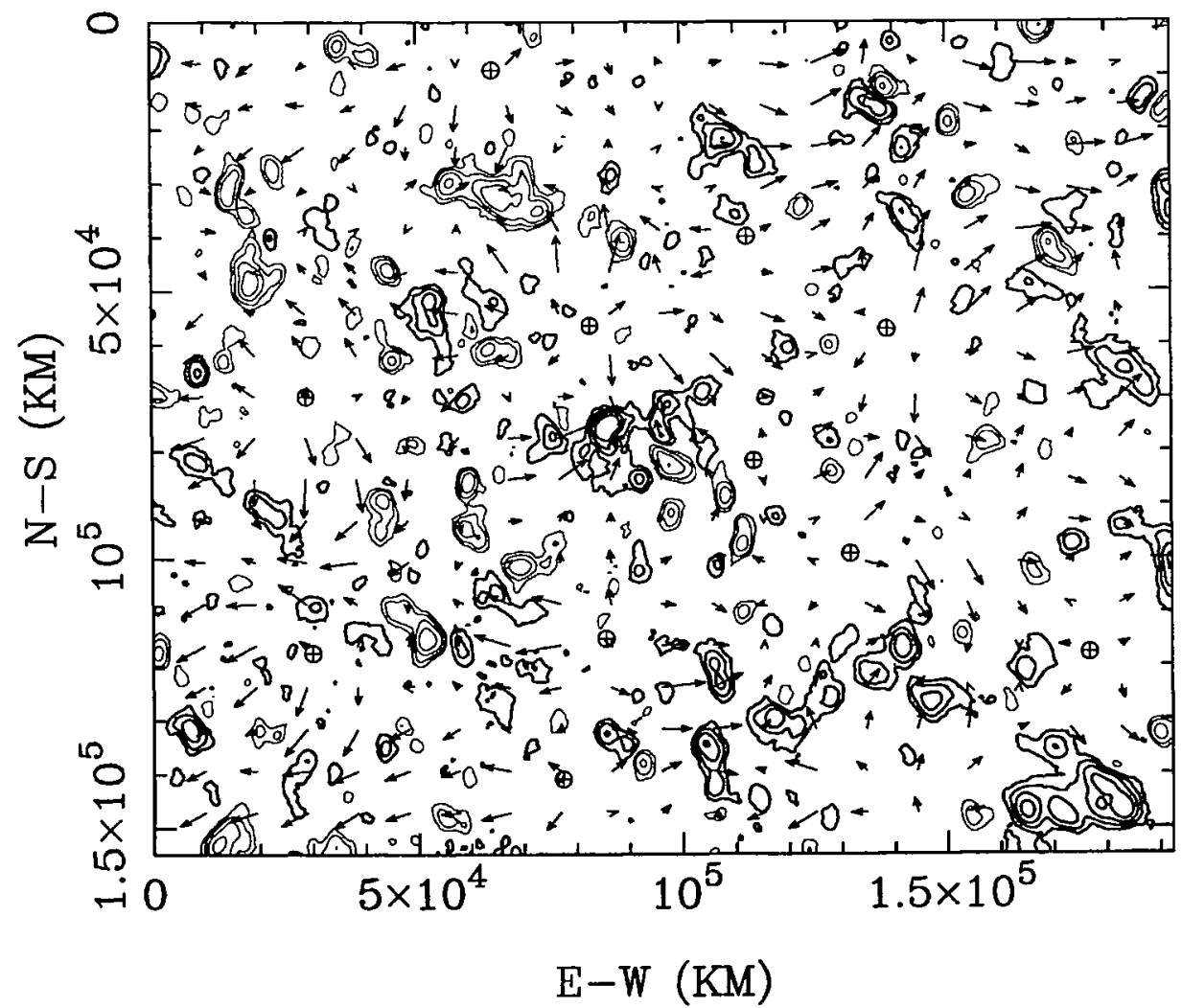

Fig. 6. Combination of the Figures 4 and 5, showing a tendency for the IN elements to move to the nearby network elements. 


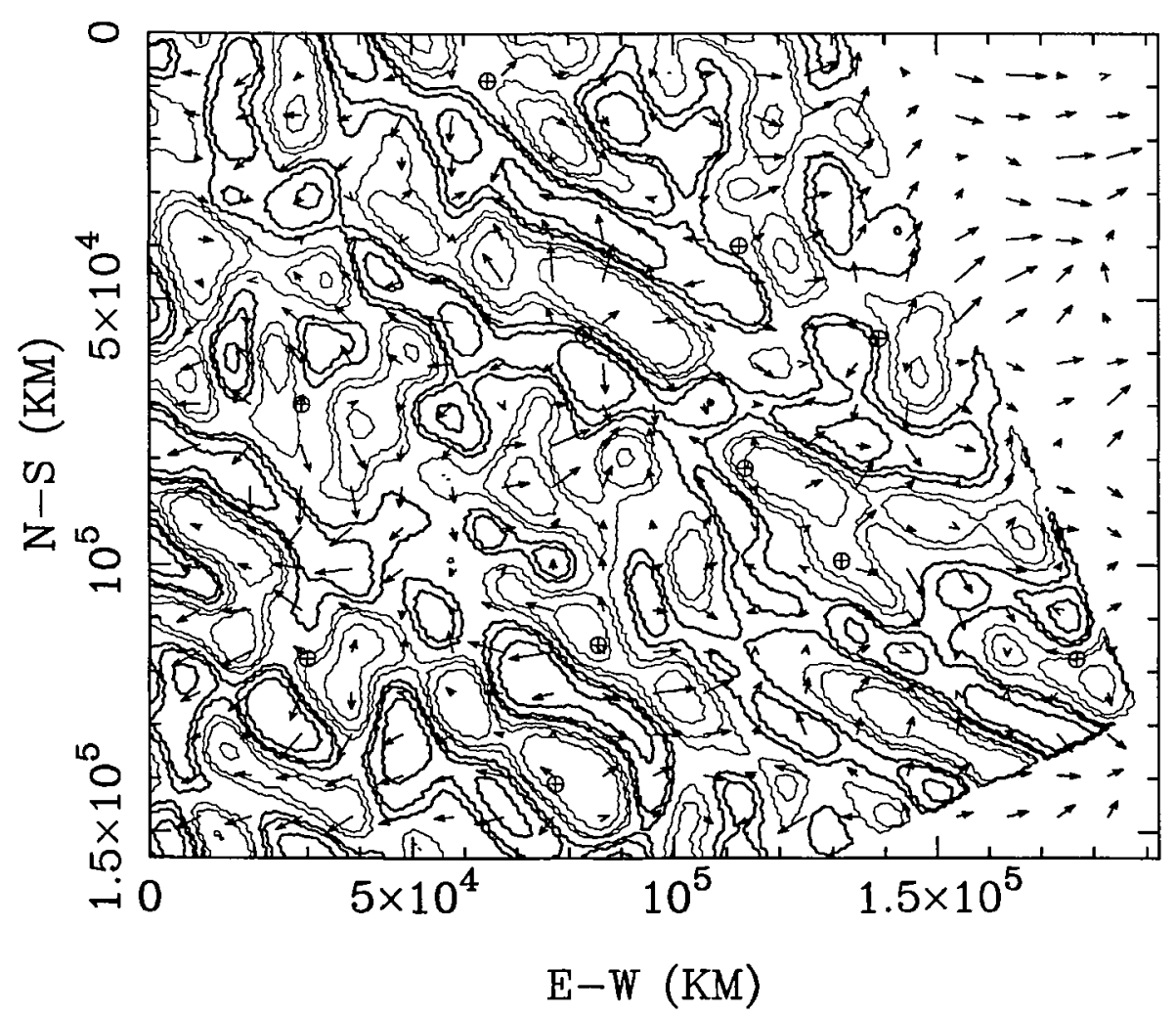

Fig. 7. Comparison of the Doppler measurement and the correlation tracking. The contours are the supergranule velocity fields, arrows represent the correlation tracking map.

elements derived from correlation tracking is roughly consistent with direct Doppler measurement.

The results of another observing run made on June 22, 1985 are shown in Figure 8. This was a quiet region near the center of the solar disk, but there was no corresponding Doppler measurement. This map gives a result similar to that for October 15, 1985.

We also studied the relationship between the amplitude of the proper motion and the mean magnetic flux density. The result is shown in Figure 9. The $X$-axis is the average magnetic flux density in Gauss of the windows defined above, and the $Y$-axis is the amplitude of the proper motion for that window. The major conclusion is: if the magnetic flux density is high, i.e., the network field, the upper limit of the proper motion speed decreases with the flux density. However, at low flux densities proper motion ranges from 0 to $0.8 \mathrm{~km} \mathrm{~s}^{-1}$. This is consistent with the results of Zirin (1985): the IN elements move fast, and network elements move slowly.

The proper motion speed of INF ranges from 0 to $0.8 \mathrm{~km} \mathrm{~s}^{-1}$; the average speed is $0.20 \mathrm{~km} \mathrm{~s}^{-1}$. If the peak of the supergranular horizontal velocity is $V_{h}$ as shown in Equation (1), the average horizontal speed over the whole cell is

$$
\langle V\rangle=\frac{\int_{0}^{2 \pi} \int_{0}^{L / 2} V_{h} \sin (2 \pi \rho / L) \rho \mathrm{d} \rho \mathrm{d} \theta}{\int_{0}^{2 \pi} \int_{0}^{L / 2} \rho \mathrm{d} \rho \mathrm{d} \theta}=\frac{2}{\pi} V_{h},
$$




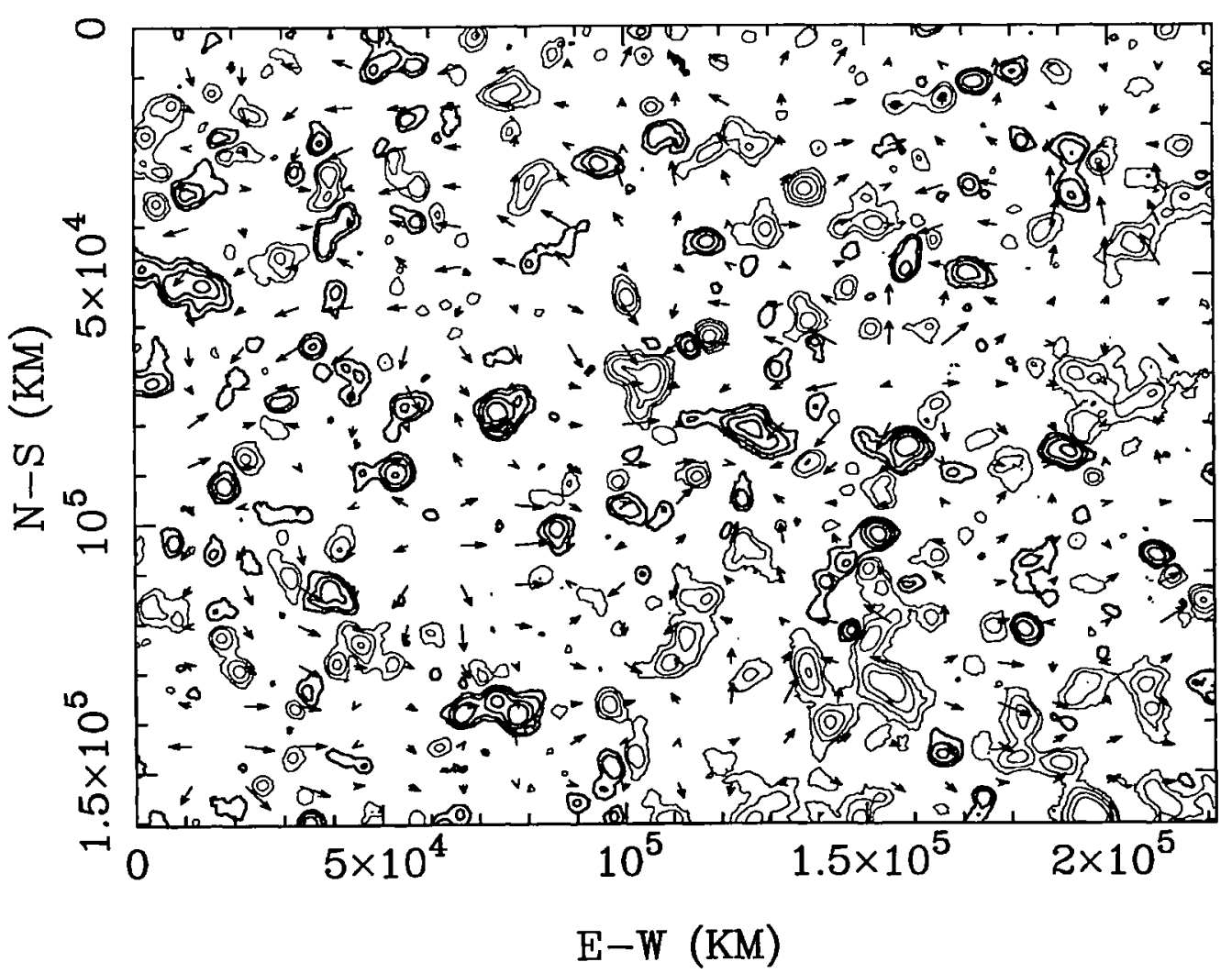

Fig. 8. The correlation tracking with the magnetogram contour map for June 22, 1985.

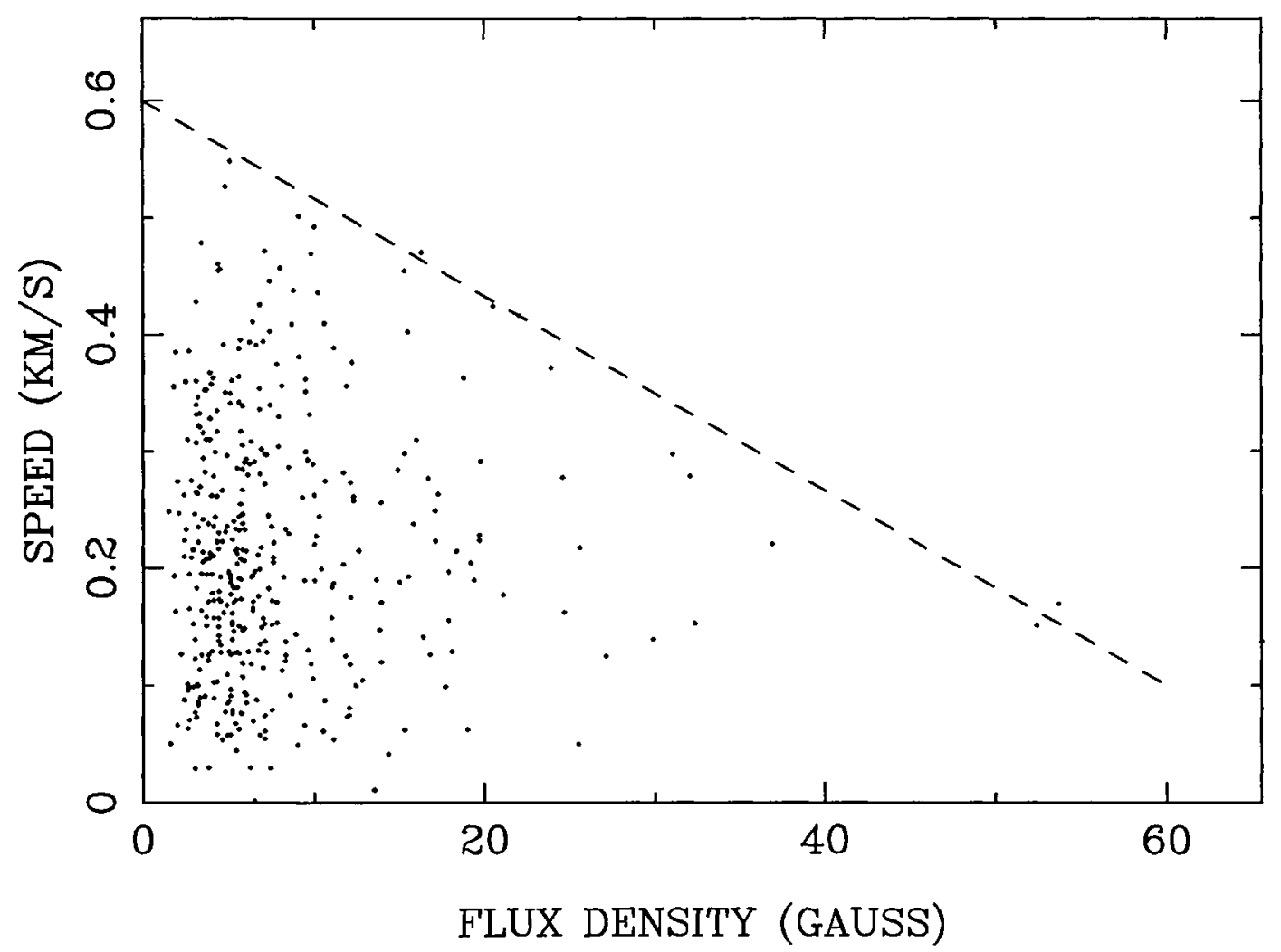

Fig. 9. The proper motion speed of magnetic elements via the average magnitude of magnetic flux density. 
so the peak speed $V_{h}$ is about $0.31 \mathrm{~km} \mathrm{~s}^{-1}$, slightly smaller than the supergranule speed obtained by previous authors (e.g., Leighton, Noyes, and Simon, 1962).

Finally, we made a numerical simulation to describe the result of the flow derived from correlation tracking. We uniformly distribute one cursor per window over the field of view. We let the cursors run with the observed 2-dimensional velocity fields and made movies based on this simulation. The motion of the cursors has an obvious cellular pattern and they are swept to areas of convergence. After 20 hours, the distribution of the cursors reaches a stable state and a network is formed. But because the IN magnetic elements are of both signs, the unipolar magnetic network cannot form this way, nor can any substantial flux element be produced.

In the steady state, most of the tracers stay in the area corresponding to the stronger magnetic elements with a few exceptions. Figure 10 shows the results of numerical



Fig. 10a.

Fig. 10. Results of numerical simulation of the correlation tracking velocity fields for October 15, 1985 . (a) is superposed on a magnetogram, (b) is superposed on a Dopplergram. 


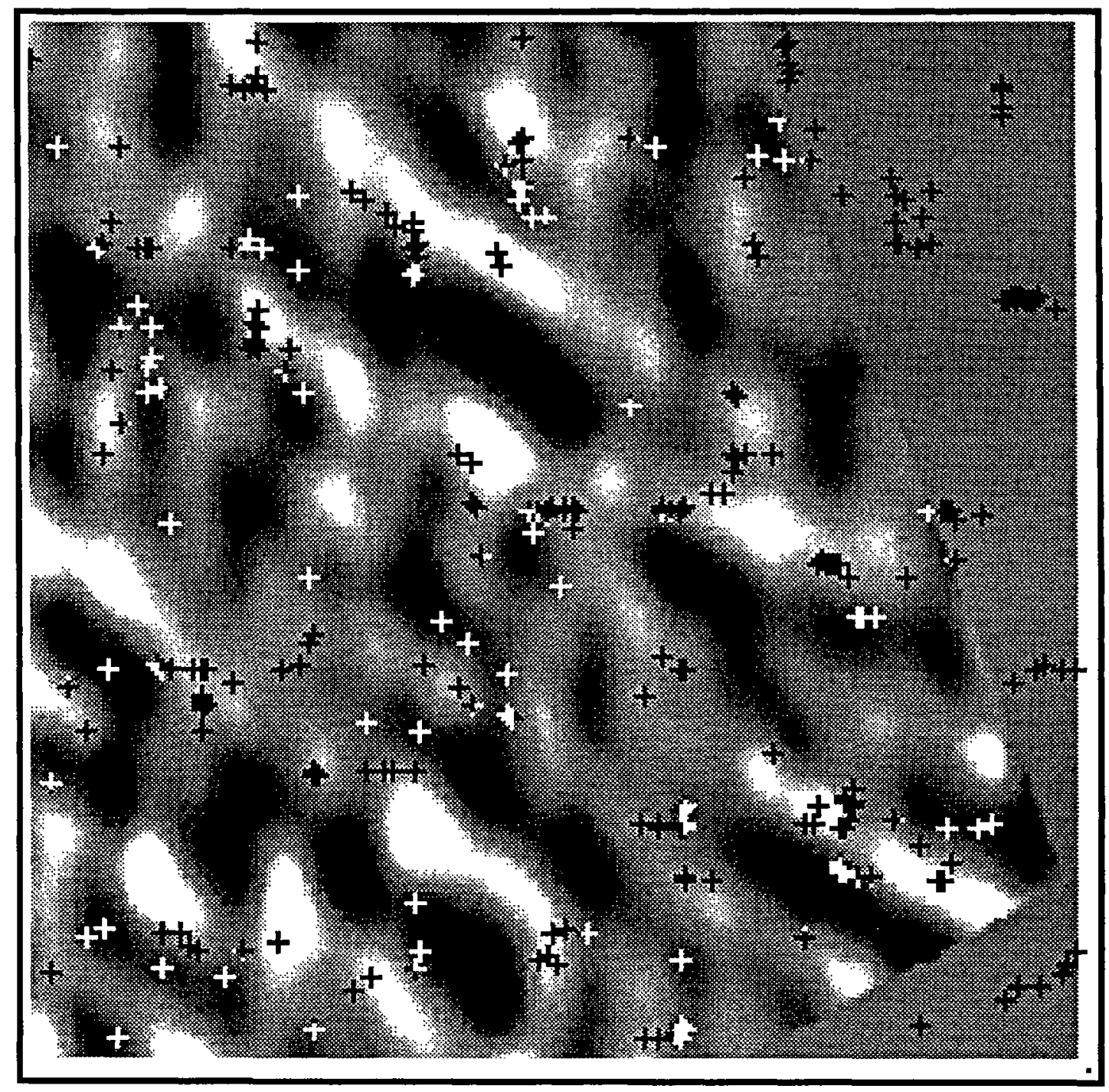

Fig. 10b.

simulation for October 15, 1985; Figure 10(a) is superposed on a magnetogram and Figure 10(b) is superposed on a Dopplergram. Figure 10(b) gives some evidence that the cursors (which represent the magnetic elements) are expelled from the strong velocity region and swept to the boundary of the supergranule cells. Figure 11 shows the result for June 22, 1985.

\section{Summary and Discussion}

By correlation tracking of intranetwork magnetic elements on Big Bear magnetograms, we have confirmed a flow pattern consistent with the supergranule velocity field. We also found a quantitative relation between the supergranule boundaries and the magnetic network using cross-correlation.

By simulation we confirmed that the intranetwork magnetic fields are swept to the boundary of the supergranule by the INF implying the magnetic network elements are 


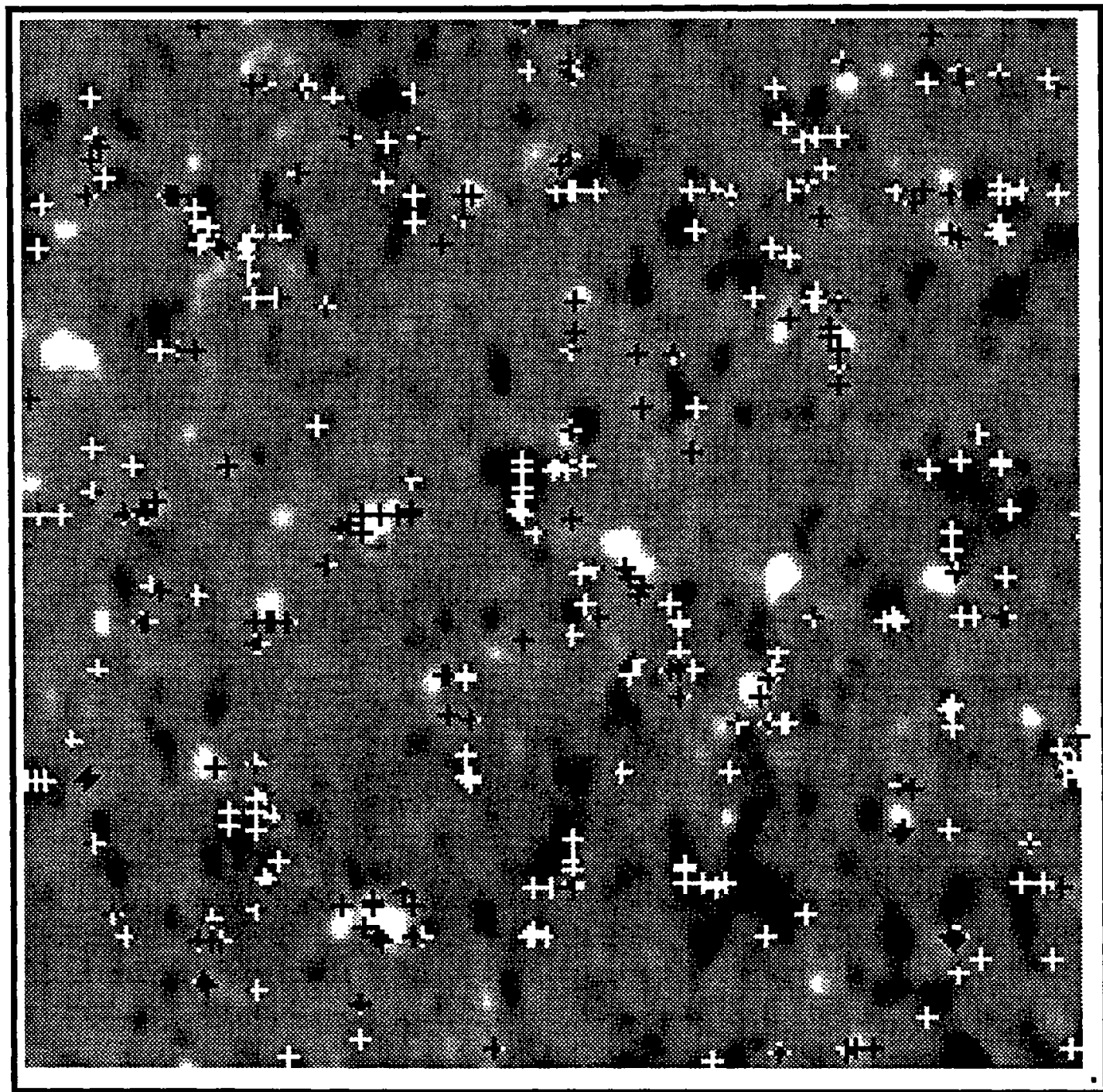

Fig. 11. Results of numerical simulation for June 22, 1985. It is superposed on a magnetogram.

confined by the supergranular flow. But this does not mean that the network elements themselves are formed by the concentration of the IN elements. The IN fields are usually of mixed polarity, each element having flux about $5 \times 10^{16}$ to $10^{17} \mathrm{Mx}$ (Livingston and Harvey, 1974; Wang, Zirin, and Shi, 1985). The network elements have typical flux $5 \times 10^{18} \mathrm{Mx}$. If the network is formed by the concentration of the intranetwork elements, 50 to 100 elements with the same polarity must be collected. The probability of one element meeting another with the same polarity is $50 \%$, so the probability that an element will meet 50 to 100 other elements with the same polarity is essentially zero.

We studied the formation and disappearance of the network elements from the VMG movies and found that of the 50 network elements that existed at the beginning of the observation, eight cancelled with other opposite polarity fields, two fragmented to little elements, and the rest of them retained their identity. Also we found nine new elements formed from ephemeral regions, and two from the concentration of diffuse fields. To 
avoid bias in selecting the ephemeral regions and cancelling features, we ran the movie forward to study the disappearance of old magnetic flux elements, and backward to study the emergence of the new flux. Thus the network is not formed by the supergranule flow, but the concentration of the network may be maintained. The detail of the magnetic flux budget and the properties of the ER and cancelling features will be discussed in another paper.

Although in general the motion of the magnetic fields follows the supergranule pattern, some flux tubes with larger flux may move under its buoyancy force. The ephemeral regions separate at about $0.6 \mathrm{~km} \mathrm{~s}^{-1}$ (Zirin, 1985), regardless of the local flow.

We should point out that the random rectilinear motion of IN elements, which cannot pass through the network, will invariably lead to the result of a simulated accumulation near the network. But the fact that most of the elements we followed did move toward the cell boundaries supports the idea that the IN elements follow the supergranular flow.

The fact that the elements follow the supergranular flow is in itself interesting. If the IN elements were shallow, they would be linked by field lines to other elements of the opposite polarity which should be seen to move with them. Instead we see individual elements of one sign or the other, which suggests that the elements are the intersection of fairly deep lines of force with the surface. It is also of interest that the moving elements show no random perturbations as might be due to exploding granules.

\section{Acknowledgements}

We are indebted to Dr S. F. Martin for many helpful comments, discussion and suggestions. We wish to thank the Big Bear staff for the support in observation, Dr B. Popp for his computer programs and J. Nenow for processing films. Also we are grateful for the assistance from Kitt Peak Observatory, especially Drs J. Harvey and K. Harvey for their valuable suggestions and F. Recely for his help in observation. This work is supported by NSF under grant ATM-8513577 and NASA under grant NGL 05002034.

\section{References}

Chou, D. and Wang, H.: 1987, Solar Phys. 110, 81.

Clark, A., Jr.: 1968, Solar Phys. 4, 386.

Galloway, D. J. and Weiss, N. O.: 1981, Astrophys. J. 243, 945.

Giovanelli, R. A.: 1980, Solar Phys. 67, 211.

Hart, A. B.: 1954, Monthly Notices Roy. Astron. Soc. 114, 2.

Hart, A. B.: 1956, Monthly Notices Roy. Astron. Soc. 116, 38.

Leighton, R. B., Noyes, R. W., and Simon, G. W.: 1962, Astrophys. J. 135, 474.

Livi, S. H. B., Wang, J., and Martin, S. F.: 1985, Australian J. Phys. 38, 855.

Livingston, W. C. and Harvey, J.: 1975, AAS Bull. 7, 346.

Livingston, W. C., Harvey, J., Pierce, A. K., Schrage, D., Gillespie, B. Simmons, J., and Slaughter, C.: 1976a, Appl. Optics 15, 33.

Livingston, W. C., Harvey, J., Slaughter, C. and Trumbo, D.: 1976b, Appl. Optics 15, 40.

Martin, S. F.: 1983, BBSO Preprint No. 228.

Martin, S. F.: 1987, Presentation on Solar Cycle Workshop, Lake Tahoe. 
Martin, S. F., Livi, S. H. B., and Wang, J.: 1985, Australian J. Phys. 38, 929.

Meyer, F., Schmidt, H. U., and Simon, G. W.: 1979, Astron. Astrophys. 76, 35.

Mosher, J. M.: 1976, BBSO Preprint No. 159.

Parker, E. N.: 1982, Astrophys. J. 256, 746.

Schmidt, H. U., Simon, G. W., and Weiss, N. O.: 1985, Astron. Astrophys. 148, 191.

Simon, G. W. and Leighton, R. B.: 1964, Astrophys. J. 140, 1120.

Shi, Z., Wang, J., and Patterson, A.: 1985, BBSO Preprint No. 257.

Spruit, H. C.: 1981, Astron Astrophys. 98, 155.

Title, A. M., Tarbell, T. D., and SOUP Team: 1987, AAS Bull. 18, 992.

Wang, H. and Zirin, H.: 1988a, in preparation.

Wang, H. and Zirin, H.: 1988b, in preparation.

Wang, J., Zirin, H. and Shi, Z.: 1985, Solar Phys. 98, 241.

Zirin, H.: 1985, Australian J. Phys. 38, 961.

Zirin, H.: 1987, Solar Phys. 110, 101. 